\title{
QUASI-POLYNOMIAL SYSTEM \\ REPRESENTATION FOR THE ANALYSIS AND CONTROL OF NONLINEAR SYSTEMS
}

\author{
A. Magyar ${ }^{*, * *}$ G. Szederkényi ${ }^{*}$ K. M. Hangos ${ }^{*, * *}$ \\ * Computer and Automation Research Institute HAS, \\ H-1518 Budapest P.O. Box 63, Hungary, \\ fax: +3614667503,e-mail:amagyar@scl.sztaki.hu \\ ** Dept. of Computer Science, University of Veszprém, \\ H-8200 Veszprém, Egyetem u. 10, Hungary
}

\begin{abstract}
The application of quasi-polynomial system representation for the analysis and control of a wide class of smooth nonlinear systems is proposed in this paper. The analysis part includes the global stability analysis of the open loop system and that of the zero dynamics. It is shown that the stabilizing controller design with monomial feedback structure leads to the feasibility of bilinear matrix inequalities. The methods are illustrated on simple examples. Copyright $(2005$ IFAC
\end{abstract}

Keywords: nonlinear systems, quasi-polynomial systems, stability, zero dynamics, stabilizing feedback controllers

\section{INTRODUCTION}

The class of quasi-polynomial (QP) systems plays an increasingly important role in the modelling of dynamical systems since the majority of smooth nonlinear systems occurring in practice can be easily transformed to QP form (Hernández-Bermejo et al., 1998). At the same time, the stability properties of QP systems have been intensively studied recently (Figueiredo et al., 2000), (HernándezBermejo, 2002).

On the other hand, some computationally effective numerical methods have been developed lately, that allow us to practically perform the stability analysis of QP systems (Figueiredo et al., 2003).

\footnotetext{
1 The support of the Hungarian Scientific Research Fund through grants T042710, F046223 is gratefully acknowledged. The second author acknowledges the support of the Bolyai János Research Scholarship of the Hungarian Academy of Sciences.
}

To the best of the authors' knowledge no one has tried to use the above mentioned theoretical and numerical tools in the framework of nonlinear control systems. This paper aims to show that the QP description of dynamical systems can offer practically feasible design methods in this challenging field.

\section{BASIC NOTIONS}

In this section some basic concepts of quasipolynomial (QP) and Lotka-Volterra (LV) systems (Hernández-Bermejo and Fairén, 1997) are introduced. Basic notions on linear- and bilinear matrix inequalities are also presented here.

\subsection{Quasi-Polynomial Models}

Quasi-polynomial models are sets of ODEs of the following form 


$$
\dot{y}_{i}=y_{i}\left(L_{i}+\sum_{j=1}^{m} A_{i j} \prod_{k=1}^{n} y_{k}^{B_{j k}}\right)
$$

where $y \in \operatorname{int}\left(\mathbf{R}_{+}^{n}\right), A \in \mathbf{R}^{n \times m}, B \in \mathbf{R}^{m \times n}, L_{i} \in$ $\mathbf{R}, i=1, \ldots, n$. Furthermore, $L=\left[\begin{array}{lll}L_{1} & \ldots & L_{n}\end{array}\right]^{T}$. Let us denote the equilibrium point of interest of (1) as $y^{*}=\left[\begin{array}{llll}y_{1}^{*} & y_{2}^{*} & \ldots & y_{n}^{*}\end{array}\right]^{T}$. Without the loss of generality we can assume that $\operatorname{Rank}(A)=$ $\operatorname{Rank}(B)=n$ and $m \geq n$.

The above family of models is split into classes of equivalence (Hernández-Bermejo and Fairén, 1997) according to the values of the products $M=B \cdot A$ and $N=B \cdot L$. The Lotka-Volterra form gives the representative elements of these classes of equivalence. If $\operatorname{Rank}(B)=n$, then the set of ODEs in (1) can be embedded into the following $m$-dimensional set of equations, which is a classical Lotka-Volterra model:

$$
\dot{z}_{j}=z_{j}\left(N_{j}+\sum_{i=1}^{m} M_{j i} z_{i}\right), \quad j=1, \ldots, m
$$

where each $z_{j}$ represents a so called quasimonomial:

$$
z_{j}=\prod_{k=1}^{n} y_{k}^{B_{j k}}, \quad j=1, \ldots, m .
$$

\subsection{Rewriting non QP models into QP-form}

A set of nonlinear ODEs can be embedded to QPform if the non-QP elements are multiplicative functions $f$ appearing in the QP-terms and a QPtype ODE can be found such that $f$ is a solution of it (Hernández-Bermejo and Fairén, 1995).

The embedding is performed by introducing a new auxiliary variable $x$ for each non-QP function $f$ which is in the simplest case $x=f$. One can differentiate this algebraic equation in order to arrive at a new ODE in QP-form that completes the embedded QP-ODE model.

It is important to note that the embedding is not unique, because we can choose the new variables in a different, more complicated way as compared to $x=f$.

\subsection{Linear and bilinear matrix inequalities}

A linear matrix inequality (LMI) is a convex constraint which can be expressed in the form

$$
F(x)=F_{0}+F_{1} x_{1}+\cdots+F_{n} x_{n}>0
$$

where

- $F_{0}, F_{1}, \cdots, F_{n}$ are given symmetric matrices

- $\left(x_{1}, \cdots, x_{n}\right)^{T} \in R^{n}$ is the vector of decision variables
Furthermore, the inequality symbol in (4) stands for the positive definiteness of $F$. If we allow the equality in (4), then we talk about a nonstrict LMI. LMIs are solved e.g. with the so called Ellipsoid algorithm (Gahinet et al., 1995) which has polynomial complexity. There are efficient software tools for solving LMIs. One of them is the MATLAB ${ }^{\circledR}$ LMI Control Toolbox (Scherer and Weiland, 2000) but there are other useful instruments, too (Figueiredo et al., 2003).

A bilinear matrix inequality (BMI) is a diagonal block composed of $q$ matrix inequalities of the following form

$$
\begin{gathered}
G_{0}^{i}+\sum_{k=1}^{p} x_{k} G_{k}^{i}+\sum_{k=1}^{p} \sum_{j=1}^{p} x_{k} x_{j} K_{k j}^{i} \leq 0, \\
i=1, \ldots, q
\end{gathered}
$$

where $x \in \mathbf{R}^{p}$ is the decision variable to be determined and $G_{k}^{i}, k=0, \ldots, p, i=1, \ldots, q$ and $K_{k j}^{i}, k, j=1, \ldots, p, i=1, \ldots, q$ are symmetric, quadratic matrices.

The main properties of BMIs are that they are non-convex in $x$ (which makes their solution numerically much more complicated than that of linear matrix inequalities), and their solution is NPhard (VanAntwerp and Braatz, 2000). However, there exist practically applicable and efficient algorithms for BMI solution (Tuan et al., 2000), (Kocvara and Stingl, 2003).

\subsection{Stability Analysis of $Q P$ and LV models}

In this section the methods of local and global stability analysis of QP and LV models, i.e. autonomous QP and LV system models are briefly summarized. Henceforth it is assumed that $y^{*}$ is a positive equilibrium point, i.e. $y^{*} \in \operatorname{int}\left(\mathbf{R}_{+}^{n}\right)$ in the $\mathrm{QP}$ case and similarly $z^{*} \in \operatorname{int}\left(\mathbf{R}_{+}^{m}\right)$ is a positive equilibrium point in the $\mathrm{LV}$ case.

2.4.1. Global stability analysis of $L V$ models For LV systems there is a well known Lyapunov function family (Hernández-Bermejo, 2002),

(Figueiredo et al., 2000), which is in the form:

$$
V(z)=\sum_{i=1}^{m} c_{i}\left(z_{i}-z_{i}^{*}-z_{i}^{*} \ln \frac{z_{i}}{z_{i}^{*}}\right)
$$

$$
c_{i}>0, \quad i=1 \ldots m \text {. }
$$

where $z^{*}=\left(z_{1}^{*}, \ldots, z_{m}^{*}\right)^{T}$ is the equilibrium point corresponding to the equilibrium $y^{*}$ of the original $\mathrm{QP}$ system. The time derivative of the Lyapunov function (6) is:

$$
\dot{V}(z)=\frac{1}{2}\left(z-z^{*}\right)\left(C M+M^{T} C\right)\left(z-z^{*}\right)
$$

where $C=\operatorname{diag}\left(c_{1}, \ldots, c_{m}\right)$ and $M$ is the invariant characterizing the $\mathrm{LV}$ form. Therefore the 
nonincreasing nature of the Lyapunov function is equivalent to the feasibility of the following set of LMIs:

$$
\begin{aligned}
C M+M^{T} C & \leq 0 \\
C & >0
\end{aligned}
$$

where the unknown matrix is $C$, which is diagonal and contains the coefficients of (6). It is important to note that the strict positivity constraint on $c_{i}$ can be somewhat relaxed in the following way (Figueiredo et al., 2000): if the equations of the model (1) are ordered in such a way that the first $n$ rows of $B$ are linearly independent, then $c_{i}>0$ for $i=1, \ldots, n$ and $c_{j}=0$ for $j=n+1, \ldots, m$ still guarantee global stability.

It is examined and proved in (Figueiredo et al., 2000) and (Hernández-Bermejo, 2002) that the global stability of (2) with Lyapunov function (6) implies the boundedness of solutions and global stability of the original QP system (1).

\section{ZERO DYNAMICS ANALYSIS OF QP SYSTEMS}

In this section we extend the autonomous QPODE models (1) first to construct QP system models. Thereafter the conditions to have a QPform of the zero dynamics of QP systems are presented.

\subsection{QP system models}

A single-output input-affine nonlinear system model

$$
\begin{aligned}
& \dot{y}=f(y)+\sum_{i=1}^{p} g_{i}(y) u_{i} \\
& \eta=h(y)
\end{aligned}
$$

(where $y \in \mathbb{R}^{n}$ is the state vector, $u \in \mathbb{R}^{p}$ is the input and $\eta \in \mathbb{R}$ is the output) is in QP-form if all of the functions $f, g$ and $h$ are in QP-form. Then the general form of the state equation of an input-affine QP system model with $p$-inputs is:

$$
\begin{aligned}
\dot{y}_{i}= & y_{i}\left(\lambda_{0_{i}}+\sum_{j=1}^{m} A_{0_{i j}} \prod_{k=1}^{n} y_{k}^{B_{j k}}\right)+ \\
& +\sum_{l=1}^{p} y_{i}\left(\lambda_{l_{i}}+\sum_{j=1}^{m} A_{l_{i j}} \prod_{k=1}^{n} y_{k}^{B_{j k}}\right) u_{l}
\end{aligned}
$$

where

$$
\begin{gathered}
i=1, \ldots, n, \quad A_{0}, A_{l} \in \mathbf{R}^{n \times m}, \quad B \in \mathbf{R}^{m \times n}, \\
\lambda_{0}, \lambda_{l} \in \mathbf{R}^{n}, \quad l=1, \ldots, p .
\end{gathered}
$$

Table 1. Variables and parameters of the bioreactor model

\begin{tabular}{llll}
\hline$X$ & biomass concentration & & {$\left[\frac{g}{l}\right]$} \\
$S$ & substrate concentration & & {$\left[\frac{g}{l}\right]$} \\
$F$ & inlet feed flow rate & & {$\left[\frac{l}{h}\right]$} \\
$V$ & volume & 4 & {$[l]$} \\
$S_{F}$ & substrate feed concentration & 10 & {$\left[\frac{g}{l}\right]$} \\
$Y$ & yield coefficient & 0.5 & - \\
$\mu_{\max }$, & kinetic parameter & 1 & {$\left[\frac{1}{h}\right]$} \\
$K_{1}$ & kinetic parameter & 0.03 & {$\left[\frac{g}{l}\right]$} \\
$K_{2}$ & kinetic parameter & 0.5 & {$\left[\frac{l}{g}\right]$} \\
\hline
\end{tabular}

\subsection{Zero dynamics analysis}

Let us consider a SISO input-affine QP-model in the form of Eq. (10) with $p=1$ and with the simplest output $\eta=y_{i}-w^{*}$ for some $i$ and $w^{*}>0$, i.e. we want to keep the system's output at a positive constant value. Moreover, let us assume that the relative degree of the system equals one and $g_{i 1}(y)=g_{i}(y)=\prod_{j=1}^{n} y_{j}^{\gamma_{j i}}$, i.e. the input function is of quasi-monomial type. Then the output zeroing input is given in the form

$$
u(t)=-\frac{L_{f_{i}} h(y)}{L_{g_{i}} h(y)}=-\frac{f_{i}(y)}{\prod_{j=1}^{n} y_{j}^{\gamma_{j i}}}
$$

where $L_{f} h$ denotes the Lie-derivative of $h$ along $f$. It is seen that the output zeroing input above is in QP-form if $f_{i}(y)$ is in QP-form.

In order to obtain the zero dynamics, one has to substitute the input (11) to the state equation (10) to obtain an autonomous system model. It is easy to compute that the resulting zero dynamics system model will remain in QP-form with an output zeroing input in QP-form. Therefore the stability analysis of the zero dynamics can be investigated using the methods described earlier in section 2.4 .

The above result can be easily generalized to the case of outputs in quasi-monomial form.

3.2.1. A simple fermentation example Consider a simple fermentation process with nonmonotonous reaction kinetics that is described by the non-QP input-affine state-space model

$$
\begin{aligned}
\dot{X} & =\mu(S) X-\frac{X F}{V} \\
\dot{S} & =-\frac{\mu(S) X}{Y}+\frac{\left(S_{F}-S\right) F}{V} \\
\mu(S) & =\mu_{\max } \frac{S}{K_{2} S^{2}+S+K_{1}},
\end{aligned}
$$

Where the inlet feed flow rate denoted by $F$ is the manipulated input. The variables and parameters of the model together with their units and parameter values are given in Table 1 . The parameter values are taken from (Kuhlmann et al., 1998).

The investigated equilibrium point of the system is where the outlet biomass flow rate (i.e. biomass production per unit time) is maximal: 


$$
\begin{aligned}
S_{0} & =\frac{1}{2} \frac{-2 K_{1}+2 \sqrt{K_{1}^{2}+S_{F}^{2} K_{1} K_{2}+S_{F} K_{1}}}{S_{F} K_{2}+1} \\
X_{0} & =\left(S_{F}-S_{0}\right) Y
\end{aligned}
$$

By introducing a new differential variable $Z=$ $\frac{1}{K_{2} S^{2}+S+K_{1}}$ in addition to $X$ and $S$, the original system (12) can be represented in QP-form characterized by the following matrices:

$$
\begin{gathered}
A_{0}=\left[\begin{array}{ccccccc}
\mu_{\max } & 0 & 0 & 0 & 0 & 0 & 0 \\
0 & -\frac{\mu_{\max }}{Y} & 0 & 0 & 0 & 0 & 0 \\
0 & 0 & 0 & \frac{2 \mu_{\max } K_{2}}{Y} & 0 & \frac{\mu_{\max }}{Y} & 0
\end{array}\right] \\
A_{1}=\left[\begin{array}{cccccccc}
0 & 0 & 0 & 0 & 0 & 0 & 0 \\
0 & 0 & \frac{S_{F}}{V} & 0 & 0 & 0 & 0 \\
\frac{1-2 K_{2} S_{F}}{V} & 0 & 0 & 0 & \frac{2 K_{2}}{V} & 0 & -\frac{S_{F}}{V}
\end{array}\right] \\
B=\left[\begin{array}{rrr}
0 & 1 & 1 \\
1 & 0 & 1 \\
0 & -1 & 0 \\
1 & 2 & 2 \\
0 & 2 & 1 \\
1 & 1 & 2 \\
0 & 0 & 1
\end{array}\right] \lambda_{1}=\left[\begin{array}{c}
-\frac{1}{V} \\
-\frac{1}{V} \\
0
\end{array}\right]
\end{gathered}
$$

The quasi-monomials in the $\mathrm{QP}$ system model are:

$$
S Z, X Z, S^{-1}, S^{2} X Z^{2}, S^{2} Z, S X Z^{2}, Z
$$

Let us choose input of the system to be the input flowrate, $F$, and the output to be the centered substrate-concentration:

$$
y=\eta=S-S_{0}
$$

The output zeroing input can be easily computed:

$$
F=\frac{\mu_{\max } S_{0} V}{Y\left(S_{F}-S_{0}\right)} X Z
$$

If the above equations are substituted into the QP-form, the matrices $A^{\prime}, B^{\prime}$ and $\lambda^{\prime}$ characterizing the zero dynamics are the following:

$$
\begin{gathered}
A^{\prime}=\left[\begin{array}{ccc}
\mu_{\max } S_{0} & -\frac{\mu_{\max } S_{0}}{Y\left(S_{F}-S_{0}\right)} & 0 \\
0 & 0 & R
\end{array}\right], \\
B^{\prime}=\left[\begin{array}{ll}
0 & 1 \\
1 & 1 \\
1 & 2
\end{array}\right], \lambda^{\prime}=\left[\begin{array}{l}
0 \\
0
\end{array}\right],
\end{gathered}
$$

where

$$
\begin{aligned}
R= & \frac{\left(1-2 K_{2} S_{F}\right) \mu_{\max } S_{0}^{2}}{Y\left(S_{F}-S_{0}\right)}+\frac{2 \mu_{\max } K_{2} S_{0}^{2}}{Y}+ \\
& +\frac{2 K_{2} \mu_{\max } S_{0}^{3}}{Y\left(S_{F}-S_{0}\right)}+\frac{\mu_{\max } S_{0}}{Y}-\frac{\mu_{\max } S_{0} S_{F}}{Y\left(S_{F}-S_{0}\right)}
\end{aligned}
$$

Hence, the monomials of the zero dynamics are

$$
Z, \quad X Z, \quad X Z^{2}
$$

Note that the number of quasi-monomials has been drastically reduced.

In order to study the local stability of the zero dynamics, we first computed the eigenvalues of the
Jacobian of the zero dynamics at the equilibrium point $X_{0}, Z_{0}$ that are

$$
-0.8022, \quad 0
$$

Thereafter the feasibility of the LMI (8) was investigated using the LMI Toolbox in Matlab for global stability analysis. The singular perturbation technique was also applied to overcome the technical difficulty caused by the zero eigenvalues of the LV coefficient matrix. The result of the LMI is the following Lyapunov function parameter matrix with $\varepsilon=-10^{-7}$ as a perturbation parameter:

$$
C=\left[\begin{array}{ccc}
8.4341 \cdot 10^{8} & 0 & 0 \\
0 & 180.33 & 0 \\
0 & 0 & 0
\end{array}\right]
$$

Therefore the global stability of the zero dynamics is proved through the QP description. This result is in good agreement with (Szederkényi et al., 2002) where the stability of the zero dynamics was proved through nonlinear coordinatestransformations.

\section{CONTROLLER DESIGN}

The output zeroing input (11) can be viewed as a nonlinear static state feedback acting on the QPform state equation (10). If the state feedback is in QP-form then the closed-loop system will also be in QP-form and its stability can be conveniently investigated by using LMIs if the feedback parameters are known and fixed.

\subsection{The controller design problem}

Therefore, one can formulate a globally stabilizing state feedback design problem for QP systems as follows. Consider arbitrary quasi-polynomial inputs in the form:

$$
u_{l}=\sum_{i=1}^{r} k_{i l} \hat{q}_{i}, \quad l=1 \ldots, p
$$

where $\hat{q}_{i}=\hat{q}_{i}\left(y_{1}, \ldots, y_{n}\right), i=1, \ldots, r$ are arbitrary quasi-monomial functions of the state variables of (10) and $k_{i l}$ is the constant gain of the quasimonomial function $\hat{q}_{i}$ in the $l$-th input $u_{l}$. The closed loop system will also be a QP system with matrices

$$
\begin{gathered}
\hat{A}=A_{0}+\sum_{l=1}^{p} \sum_{i=1}^{r} k_{i l} A_{l}, \quad \hat{B}, \\
\lambda=\lambda_{0}+\sum_{l=1}^{p} \sum_{i=1}^{r} k_{i l} \lambda_{l} .
\end{gathered}
$$

where $k_{i l}$ is the $(i, l)$-th entry of the gain matrix $k$. Note that the number of quasi-monomials in the closed-loop system (i.e. the dimension of 
the matrices) together with the matrix $\hat{B}$ may significantly change depending on the choice of the feedback structure, i.e. on the quasi-monomial functions $\hat{q}_{i}$.

Furthermore, the LV coefficient matrix $M$ can also be expressed in the form:

$$
M=\hat{B} \cdot \hat{A}=M_{0}+\sum_{l=1}^{p} \sum_{i=1}^{r} k_{i l} M_{l}
$$

Then the global stability analysis of the closed loop system with unknown feedback gains $k_{i l}$ leads to the following bilinear matrix inequality

$$
\begin{aligned}
& M^{T} C+C M=M_{0}^{T} C+C M_{0}+ \\
& +\sum_{l=1}^{p} \sum_{i=1}^{r} k_{i l}\left(M_{l}^{T} C+C M_{l}\right)<0,
\end{aligned}
$$

where $C$ is diagonal and positive definite. The variables of the BMI are the $p \times r k_{i l}$ inputparameters and the $c_{j}, j=1, . ., \hat{m}$ parameters of the Lyapunov function. If the BMI above is feasible then there exists a globally stabilizing feedback with the selected structure.

4.1.1. Feedback structure design Clearly, the general feedback structure (16) should be specialized in order to reduce the number of quasi monomials in the closed-loop system. This can be performed by analyzing carefully the relationship between the quasi-monomials of the openloop system. Further reduction can be possibly achieved by choosing appropriate feedback gain values from the feasible set.

The following example illustrates the above approach to design globally stabilizing static QP feedback controllers.

\subsection{Example}

Let the open loop system be the following one:

$$
\begin{aligned}
\dot{x_{1}}= & x_{1}\left(-0.25 x_{1}^{-5 / 3} x_{2}^{-1}+\right. \\
& +(0.3413-0.0833 \cdot u) x_{1}^{-5 / 3} x_{2}^{-2}- \\
& -0.1666 x_{1}^{-1 / 15} x_{2}^{3 / 5}-0.4166 x_{1}^{13 / 6} x_{2}^{-3 / 2}+ \\
& \left.+0.3333 x_{1}^{-5 / 6} x_{2}^{1 / 2}\right) \\
\dot{x_{2}=} & x_{2}\left(0.0277 x_{1}^{13 / 6} x_{2}^{-3 / 2}+0.1666 x_{1}^{-2 / 3} x_{2}^{-2}+\right. \\
& +0.2592 x_{1}^{14 / 15} x_{2}^{-2 / 5}+ \\
& \left.+(0.1296 \cdot u-0.5309) x_{1}^{-1 / 15} x_{2}^{-2 / 5}\right)
\end{aligned}
$$

The system can also be given by the following quasi-monomials and system matrices:

$$
\begin{gathered}
x_{1}^{-5 / 3} x_{2}^{-1}, \quad x_{1}^{-5 / 3} x_{2}^{-2}, \quad x_{1}^{-1 / 15} x_{2}^{3 / 5}, \\
x_{1}^{13 / 6} x_{2}^{-3 / 2}, \quad x_{1}^{-5 / 6} x_{2}^{1 / 2}, \quad x_{1}^{-2 / 3} x_{2}^{-2}, \\
x_{1}^{14 / 15} x_{2}^{-2 / 5}, \quad x_{1}^{-1 / 15} x_{2}^{-2 / 5} .
\end{gathered}
$$

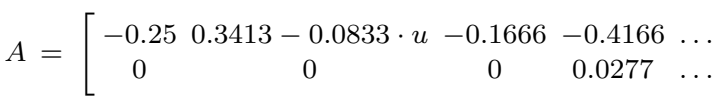

$$
\begin{aligned}
& \left.\begin{array}{ccccc}
\ldots & 0.3333 & 0 & 0 & 0 \\
\ldots & 0 & 0.1666 & 0.2592 & 0.1296 \cdot u-0.5309
\end{array}\right] \\
& B=\left[\begin{array}{cc}
-5 / 3 & -1 \\
-5 / 3 & -2 \\
-1 / 15 & 3 / 5 \\
13 / 6 & -3 / 2 \\
-5 / 6 & 1 / 2 \\
-2 / 3 & -2 \\
14 / 15 & -2 / 5 \\
-1 / 15 & -2 / 5
\end{array}\right], \lambda=\left[\begin{array}{l}
0 \\
0
\end{array}\right]
\end{aligned}
$$

The open loop system (18) has a positive equilibrium point at $x_{a}^{*}=\left[\begin{array}{ll}0.8921 & 0.7704\end{array}\right]^{T}$, which is locally stable because the eigenvalues of the Jacobian matrix in this point are: $-0.5439,-1.7824$. The open loop system has another equilibrium point at

$$
x_{b}^{*}=\left[\begin{array}{ll}
0.0380 & 1.6639
\end{array}\right]^{T} .
$$

In this point the eigenvalues of the Jacobian are: $5.9029,-1.7392$, so it is an unstable equilibrium point of the system. It can be checked that by choosing e.g. $\left[\begin{array}{ll}0.01 & 5\end{array}\right]^{T}$ as initial condition, the solution goes to infinity, therefore $x_{a}^{*}$ is not a globally stable equilibrium.

Now we try to globally stabilize the equilibrium point $x_{a}^{*}$ with the simplest (linear) monomial feedback (because with a linear static feedback of these states we expect that it will be possible to find parameter values $k_{1}$ and $k_{2}$ for which the number of monomials decreases). The feedback structure has the following form:

$$
u=-k_{1}\left(x_{1}-x_{1}^{*}\right)-k_{2}\left(x_{2}-x_{2}^{*}\right)
$$

where $\left[\begin{array}{ll}x_{1}^{*} & x_{2}^{*}\end{array}\right]^{T}=x_{a}^{*}$.

Substituting the above structure into system (18) one gets a QP system with the following quasimonomials and parameters:

$$
\begin{aligned}
& x_{1}^{-5 / 3} x_{2}^{-1}, \quad x_{1}^{-5 / 3} x_{2}^{-2}, \quad x_{1}^{-2 / 3} x_{2}^{-2}, \\
& x_{1}^{-1 / 15} x_{2}^{3 / 5}, \quad x_{1}^{13 / 6} x_{2}^{-3 / 2}, \quad x_{1}^{-5 / 6} x_{2}^{1 / 2}, \\
& x_{1}^{14 / 15} x_{2}^{-2 / 5}, \quad x_{1}^{-1 / 15} x_{2}^{-2 / 5} . \\
& A=\left[\begin{array}{cc}
-0.2500+0.0833 k_{1} & \ldots \\
0 & \cdots
\end{array}\right. \\
& \text {.. } 0.3413-0.0743 k_{1}-0.0642 k_{2} 0.0833 k_{1} \ldots \\
& \begin{array}{llll}
\ldots & 0 & 0.1666 & \ldots
\end{array} \\
& \begin{array}{lllll}
\ldots & -0.1666 & -0.4166 & 0.3333 & 0
\end{array} \\
& \text {.. }-0.1296 k_{2} \quad 0.0277 \quad 0 \quad 0.2592-0.1296 k_{1} \\
& \left.\begin{array}{cc}
\ldots & 0 \\
\ldots & -0.5309+0.1156 k_{1}+0.0998 k_{2}
\end{array}\right] \\
& B=\left[\begin{array}{cc}
-5 / 3 & -1 \\
-5 / 3 & -2 \\
-2 / 3 & -2 \\
-1 / 15 & 3 / 5 \\
13 / 6 & -3 / 2 \\
-5 / 6 & 1 / 2 \\
14 / 15 & -2 / 5 \\
-1 / 15 & -2 / 5
\end{array}\right], \lambda=\left[\begin{array}{l}
0 \\
0
\end{array}\right]
\end{aligned}
$$


It can be seen that $A$ can be written in the form:

$$
A=A_{0}+A_{1} k_{1}+A_{2} k_{2},
$$

so the design of a globally stabilizing controller may be reduced to a BMI:

$$
\begin{gathered}
=M^{T} C+C M<0, \quad \text { where } C=\operatorname{diag}\left[c_{1}, \ldots, c_{8}\right] \\
\begin{array}{c}
\left(M_{0}^{T} C+C M_{0}\right)+\left(M_{1}^{T} k_{1} C+C k_{1} M_{1}\right)+ \\
+\left(M_{2}^{T} k_{2} C+C k_{2} M_{2}\right)<0
\end{array}
\end{gathered}
$$

It can be easily checked that the following vectors are feasible solutions of (21)

$$
\begin{aligned}
& c_{1}>0, \quad c_{2}>0, \quad c_{3}=1, \\
& c_{4}=10, \quad c_{5}=2, \quad c_{6}=4, \\
& c_{7}>0, \quad c_{8}>0, \quad k_{1}=2, \quad k_{2}=3,
\end{aligned}
$$

then the monomials $x_{1}^{-5 / 3} x_{2}^{-1}, x_{1}^{-5 / 3} x_{2}^{-2}, x_{1}^{14 / 15} x_{2}^{-2 / 5}$, and $x_{1}^{-1 / 15} x_{2}^{-2 / 5}$ disappear.

The closed loop system is the following quasipolynomial system:

$$
\begin{aligned}
\dot{x}_{1}= & x_{1}\left(0.1666 x_{1}^{-2 / 3} x_{2}^{-2}-0.1666 x_{1}^{-1 / 15} x_{2}^{3 / 5}-\right. \\
& \left.-0.4166 x_{1}^{13 / 6} x_{2}^{-3 / 2}+0.3333 x_{1}^{-5 / 6} x_{2}^{1 / 2}\right) \\
\dot{x}_{2}= & x_{2}\left(0.1666 x_{1}^{-2 / 3} x_{2}^{-2}-0.3889 x_{1}^{-1 / 15} x_{2}^{3 / 5}+\right. \\
& \left.+0.0278 x_{1}^{13 / 6} x_{2}^{-3 / 2}\right)
\end{aligned}
$$

With system matrices:

$$
\begin{gathered}
A=\left[\begin{array}{cccc}
0.1666 & -0.1666 & -0.4166 & 0.3333 \\
0.1666 & -0.3889 & 0.0278 & 0
\end{array}\right] \\
B=\left[\begin{array}{cc}
-2 / 3 & 2 \\
-1 / 15 & 3 / 5 \\
13 / 6 & -3 / 2 \\
-5 / 6 & 1 / 2
\end{array}\right], \lambda=\left[\begin{array}{l}
0 \\
0
\end{array}\right]
\end{gathered}
$$

The system (22) now has only $x_{a}^{*}$ as an equilibrium point in the positive orthant, for which the eigenvalues of the Jacobian matrix are: $\lambda_{1}=$ $-1.2596, \lambda_{2}=-0.9552$.

However, the stability of $x_{a}^{*}$ is now global with the Lyapunov function of the form (6) with parameters $c_{1}, \ldots, c_{8}$.

\section{CONCLUSIONS AND FUTURE WORK}

A QP description has been proposed and found to be particularly useful for constrained (zero) dynamics analysis of relative degree 1 input-affine nonlinear systems with quasi-monomial type inputs and outputs.

It has also been shown that the globally stabilizing controller design problem with quasi-polynomial feedback structure for QP systems having relative degree 1 leads to the feasibility of a bilinear matrix inequality where the unknowns to be determined are the parameters of the Lyapunov function of the closed loop system and the constant coefficients of the monomials in the feedback law.
The feasibility of the proposed methods and tools are demonstrated using illustrative simple examples.

The development of a systematic method for feedback structure selection based on the present results is the target of future research.

\section{REFERENCES}

Figueiredo, A., I. M. Gleria and T. M. Rocha Filho (2000). Boundedness of solutions and Lyapunov functions in quasi-polynomial systems. Physics Letters A 268, 335-341.

Figueiredo, A., I. M. Gleria and T. M. Rocha Filho (2003). A numerical method for the stability analysis of quasi-polynomial vector fields. Nonlinear Analysis 52, 329-342.

Gahinet, P., A. Nemirovski, A. J. Laub and M. Chilali (1995). LMI Control Toolbox Users Guide. The MathWorks, Inc.

Hernández-Bermejo, B. (2002). Stability conditions and Liapunov functions for quasipolynomial systems. Applied Mathematics Letters 15, 25-28.

Hernández-Bermejo, B. and V. Fairén (1995). Nonpolynomial vector fields under the LotkaVolterra normal form. Physics Letters A 206, 31-37.

Hernández-Bermejo, B. and V. Fairén (1997). Lotka-volterra representation of general nonlinear systems. Math. Biosci. 140, 1-32.

Hernández-Bermejo, B., V. Fairén and L. Brenig (1998). Algebraic recasting of nonlinear ODEs into universal formats. J. Phys. A, Math. Gen. 31, 2415-2430.

Kocvara, M. and M. Stingl (2003). A code for convex nonlinear and semidefinite programming. Optimization Methods and Software 8, 317333.

Kuhlmann, Ch., I. D. L. Bogle and Z. S. Chalabi (1998). Robust operation of fed batch fermenters. Bioprocess Engineering 19, 53-59.

Scherer, C. and S. Weiland (2000). Linear Matrix Inequalities in Control. DISC. http://www.er.ele.tue.nl/sweiland/lmi.pdf.

Szederkényi, G., N.R. Kristensen and S.B. Joergensen (2002). Nonlinear analysis and control of a continuous fermentation process. Computers and Chemical Engineering 26, 659670.

Tuan, H.D., P. Apkarian and Y. Nakashima (2000). A new Lagrangian dual global optimization algorithm for solving bilinear matrix inequalities. International Journal of Robust and Nonlinear Control 10, 561-578.

VanAntwerp, J.G. and R.D. Braatz (2000). A tutorial on linear and bilinear matrix inequalities. Journal of Process Control 10, 363-385. 